\title{
Evaluation of rationality in prescribing, adherence to treatment guidelines, and direct cost of treatment in intensive cardiac care unit: A prospective observational study
}

\author{
Rohan P. Christian, Devang A. Rana, Supriya D. Malhotra, Varsha J. Patel
}

Background: Cardiovascular diseases (CVDs) remain the most common cause of sudden death. Hence, appropriate drug therapy in intensive cardiac care unit (ICCU) is crucial in managing cardiovascular emergencies and to decrease morbidity and mortality. Objective: To evaluate prescribing pattern of drugs and direct cost of therapy in patients admitted in ICCU. Materials and Methods: Patients admitted in ICCU of a tertiary care teaching hospital were enrolled. Demographic data, clinical history, and complete drug therapy received during their stay in ICCU were noted. Data were analyzed for drug utilization pattern and direct cost of treatment calculated using patient's hospital and pharmacy bills. Rationality of therapy was evaluated based on American College of Cardiology/American Heart Association (ACC/AHA) guidelines. Result: Data of 170 patients were collected over 2 months. Mean age of patients was $54.67 \pm 13.42$ years. Male to female ratio was 2.33:I. Most common comorbid condition was hypertension 76 (44.7\%). Most common diagnosis was acute coronary syndrome (ACS) 49.4\%. Mean stay in ICCU was $4.42 \pm 1.9$ days. Mean number of drugs prescribed per patient was II $.43 \pm 2.85$.Antiplatelet drugs were the most frequently prescribed drug group (86.5\%). Mean cost of pharmacotherapy per patient was ₹270I.24 \pm 3 I I I.94. Mean direct cost of treatment per patient was ₹ I0564.74 \pm I4968.70. Parenteral drugs constituted $42 \%$ of total drugs and $90 \%$ of total cost of pharmacotherapy. Cost of pharmacotherapy was positively correlated with number of drugs $(P=0.000)$ and duration of stay $(P=0.027)$. Conclusion: Antiplatelet drugs were the most frequently prescribed drug group. Mean number of drugs per encounter were high, which contributed to the higher cost of pharmacotherapy.ACC/AHA guidelines were followed in majority of the cases.

Keywords: Acute coronary syndrome, direct treatment cost, drug utilization, intensive cardiac care unit

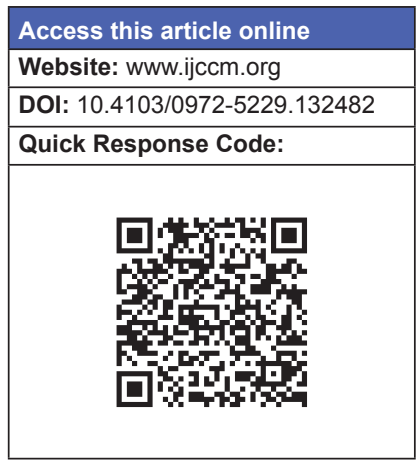

\section{Introduction}

Industrialization, urbanization, and associated lifestyle changes lead to increase prevalence of obesity, type-2 diabetes mellitus, and metabolic syndrome, which

\section{From:}

Department of Pharmacology, Smt. Nathiba Hargovandas Lakhmichand

Municipal Medical College, Ahmedabad, Gujarat, India

\section{Correspondence:}

Dr. Devang A. Rana, Department of Pharmacology,

Smt. Nathiba Hargovandas Lakhmichand Municipal Medical College,

Ellisbridge, Ahmedabad - 380 006, Gujarat, India.

E-mail: devangandu@gmail.com are important risk factors for atherosclerosis and also for cardiovascular diseases (CVDs). There is a global rise in CVDs in the $20^{\text {th }}$ and $21^{\text {st }}$ centuries among all races, ethnic groups, and cultures. CVDs are highly prevalent diseases, diagnosed in 80 million adults, i.e., one-third of the adult population. CVDs remain the most common cause of death, accounting for 35\% of all deaths, i.e. almost one million deaths each year. Approximately one-fourth of these deaths are sudden. ${ }^{[1,2]}$ More than $80 \%$ of the deaths occurred in low and middle income countries. In India, CVDs are the largest cause of mortality, accounting for around one-fourth of all deaths 
in 2008. A more worrying fact is that the incidences of CVDs have gone up significantly to $24.8 \%$ for productive age group between the 25 and 69 years. Among CVDs, $95 \%$ of prevalence and $85 \%$ of all CVDs-related deaths are contributed by coronary heart diseases (CHDs) in $2004 .{ }^{[3]}$ Indians have genetic predisposition for higher and earlier risk of CHDs than different races all over the world ${ }^{[4,5]}$ In addition, rapid socioeconomic growth in developing countries like India increases exposure to risk factors for developing CHDs like diabetes, dyslipidemia, hypertension, and smoking. ${ }^{[6,7]}$

Acute coronary syndromes (ACS) and acute myocardial infarction, acute decompensated heart failure (HF), arrhythmias, and various other cardiac conditions are emergencies that require specialized equipped intensive cardiac care unit (ICCU) setup to perform lifesaving emergency interventions like fibrinolysis, primary percutaneous coronary intervention (PCI), and defibrillation. Certain drugs like antiplatelet, anticoagulants, and fibrinolytics are needed to be administered at the earliest to these critical patients. There are many drugs prescribed in a single patient simultaneously in ICCU with the aim of maximizing efficacy in a particular condition. ${ }^{[8]}$ Prescribing rationally in ICCU is important to minimize chances of drug interactions, adverse drug reactions, and unduly high cost of treatment. Rationality of drug prescriptions can be analyzed based on recent American Heart Association/ American College of Cardiology (AHA/ ACC) guidelines in various cardiovascular morbidities. ${ }^{[9-11]}$

Pharmacoepidemiology may be drug-oriented, emphasizing the safety and effectiveness of individual drugs or groups of drugs, or utilization-oriented, aiming to improve the quality of drug therapy through pedagogic intervention. Drug utilization study is an essential part of pharmacoepidemiology, and it provides insight into prescribing pattern, prescription quality, determinants, and outcomes of drug use. ${ }^{[12]}$

To our knowledge, there are limited numbers of studies from India in this direction. ${ }^{[13,14]}$ Hence, this study was aimed to evaluate prescribing pattern and direct cost of therapy in patients admitted in ICCU.

\section{Materials and Methods}

A prospective observational study was carried out in ICCU of a tertiary teaching care hospital after obtaining approval from institutional ethics committee. Study was carried out over a period of 2 months $-1^{\text {st }}$ August to $30^{\text {th }}$ September, 2012. All the patients (including moribund patients) admitted in the ICCU during this period were enrolled in the study after obtaining a written informed consent of patient or relative. Demographic data like name, age, sex, address, socioeconomic class were recorded on the case record form. Patients' clinical data including diagnosis, detailed history of illness, past history, family history were noted. The details of drug therapy including the drug prescribed, dose, frequency, and duration of the treatment were noted in the case record form.

Prescribed drugs were analyzed for inclusion in National List of Essential Medicines (NLEM), India, 2011 and Essential Drug List (EDL) of World Health Organization (WHO), 2011. ${ }^{[15,16]}$ Rationality of therapy was evaluated based on class of recommendation by referring to ACC/AHA guidelines. ${ }^{[9-11]}$ Direct cost of treatment was calculated using patient's hospital and pharmacy bills. The cost of drugs was obtained from commercial publications like Indian Drug Review 2012 and current index of medical specialities (CIMS) online. ${ }^{[17,18]}$

\section{Statistical analysis}

All the data were recorded in Microsoft Excel 2010 spread sheet ${ }^{\circledast}$. Analyses were done using Statistical Package for the Social Sciences (SPSS) version 21.0 ${ }^{\circledR}$. A $P<0.05$ was considered statistically significant. We used unpaired $t$-test to compare between ACS with complications and without complications. We used Pearson's coefficient to correlate between number of drugs, stay in days, and cost of pharmacotherapy.

\section{Results}

\section{Demographic and clinical characteristics}

A total of 170 patients were included over duration of 2 months. Mean age of patients was $54.67 \pm 13.42$ years. Most of the patients were male 119 (70\%). Majority of the patients belonged to age group of 51-70 years, which comprised around $60 \%$ of the total patients.

Hypertension 76 (44.7\%), ischemic heart disease $75(44.1 \%)$, and type-2 diabetes mellitus 49 (28.82\%) constitute majority of the comorbid conditions. Most of the patients presented with chest pain $70.6 \%$ and breathlessness 87 (51.2\%). Most common addiction was smoking in $78(45.9 \%)$ patients. Mean stay in ICCU was $4.42 \pm 1.9$ days (range 1-12).

\section{Morbidity pattern}

Distribution of morbidity pattern is shown in Figure 1. Most common diagnosis was ACS in 84 (49.4\%) patients with mean age of $55.80 \pm 11.22$ years. Of 84 ACS patients, 22 (26.2\%) developed complications like 


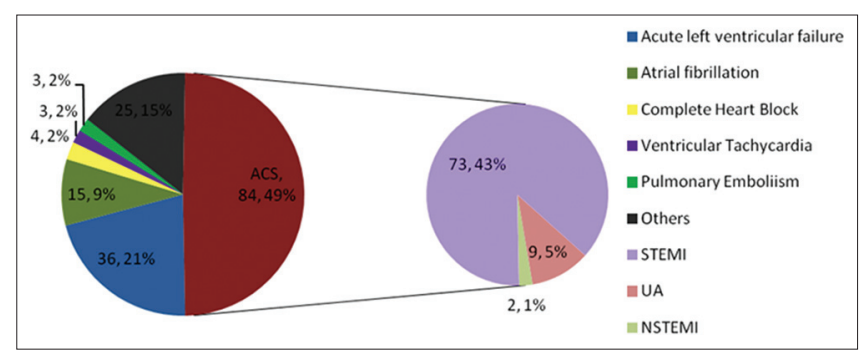

Figure I: Morbidity pattern in ICCU $(n=170)$, ICCU: Intensive cardiac care unit; ACS: Acute coronary syndrome; STEMI: ST elevation myocardial infarction; UA: Unstable angina; NSTEMI: Non-ST elevation myocardial infarction

acute decompensated HF, complete heart block, partial atrioventricular block (AV)-block, atrial fibrillation, and so forth. Majority of the complications were seen in ST elevation myocardial infarction (STEMI) patients, i.e. $20(37.7 \%)$ of 53 STEMI patients. Rest two patients with complications were of unstable angina. Table 1 shows comparison of variables in complicated and uncomplicated ACS patients $(n=84)$.

Second most common diagnosis was acute decompensated left ventricular failure (LVF) in $36(21.2 \%)$ patients. Atrial fibrillation $15(8.8 \%)$ was the third most commonly diagnosed CVD.

\section{Prescribing pattern}

A total of 1943 drugs were prescribed to 170 patients. Mean number of drugs prescribed per patient was $11.43 \pm 2.85$ (range 3-20). Antiplatelet drugs were the most frequently prescribed drug group in $86.5 \%$ of the patients. Aspirin 147 (86.5\%) was most frequently prescribed drug followed by clopidogrel 145 (85.3\%). Table 2 shows frequently prescribed drugs $(n=170)$.

\section{Drug use in acute coronary syndrome}

Aspirin and clopidogrel were prescribed to all 84 patients suffering from ACS. Streptokinase was used for thrombolysis in more than two-third patients (67.1\%) of STEMI. Unfractionated heparin (UFH) was used in $85.7 \%$ ACS patients, and low molecular weight heparin (LMWH) was used in $4.8 \%$ ACS patients. Dopamine and dobutamine were used in $16(19.1 \%)$ and nine $(10.7 \%)$ ACS patients, respectively. Glycoprotein $\mathrm{IIb} / \mathrm{III}$ a receptor antagonists were not used in any ACS patients. Tramadol was used in $76(44.7 \%)$ of the total patients and $63(75 \%)$ patients of ACS.

Figures 2 and 3 show use of drugs in STEMI and unstable angina/non-STEMI UA/NSTEMI patients, respectively, using class of recommendation given by ACC/AHA guidelines. ${ }^{[9-11]}$ Apart from use of calcium channel blockers in four patients and intravenous

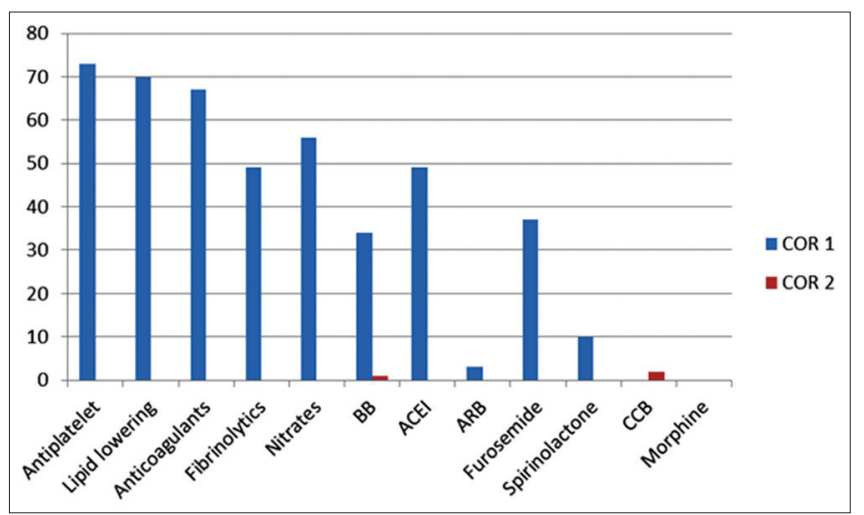

Figure 2: Use of drugs based on ACC/ACS guideline-class of recommendation in STEMI patients $(n=73)$. ACC: American College of Cardiology; ACS: Acute coronary syndrome; STEMI: ST elevation myocardial infarction; COR: Class of recommendation

Table I: Comparison of variables in complicated and noncomplicated ACS patients

\begin{tabular}{lccc}
\hline Variables & $\begin{array}{c}\text { ACS without } \\
\text { complications }\end{array}$ & $\begin{array}{c}\text { ACS with } \\
\text { complications }\end{array}$ & P value* \\
\hline Age & $53.98 \pm 10.59$ & $60.91 \pm I I .63$ & 0.012 \\
Hospital stay & $3.84 \pm 1.78$ & $6.32 \pm 1.89$ & $<0.0001$ \\
Number of drugs & $11.81 \pm 2.17$ & $13.14 \pm 2.61$ & 0.0217 \\
Cost of pharmacotherapy & $3738.84 \pm 4329.87$ & $3841.05 \pm 2024.70$ & 0.9156 \\
Total cost & $7159.73 \pm 8189.40$ & $8152.41 \pm 3366.32$ & 0.5834 \\
\hline
\end{tabular}

Unpaired $t$ test was used for comparison between two groups, *P value was considered less than 0.05 as significant, ACS: Acute coronary syndrome. $n=84$

Table 2: Most frequently prescribed drugs

\begin{tabular}{|c|c|c|c|c|}
\hline Drug group & Drug & $\begin{array}{l}\text { ATC } \\
\text { code }\end{array}$ & $\begin{array}{l}\text { Number of } \\
\text { patients (\%) }\end{array}$ & Percentage \\
\hline \multirow[t]{2}{*}{ Antiplatelet } & Aspirin & B0IAC06 & 147 (86.47) & 86.47 \\
\hline & Clopidogrel & BOIACO4 & 145 (85.29) & 85.29 \\
\hline PPI & Pantoprazole & $\mathrm{A} 02 \mathrm{BCO} 2$ & $136(80.00)$ & 80.00 \\
\hline $\begin{array}{l}\text { HMG Co-A } \\
\text { reductase inhibitor }\end{array}$ & Atorvastatin & CIOAA05 & $129(75.88)$ & 75.88 \\
\hline $\begin{array}{l}\text { 5-HT3 receptor } \\
\text { antagonist }\end{array}$ & Ondansetron & A04AA0I & $123(72.35)$ & 72.35 \\
\hline Anticoagulant & Heparin & BOIABOI & $105(6 \mid .76)$ & 61.76 \\
\hline Loop diuretic & Furosemide & C03CAOI & IOI (59.4I) & 59.41 \\
\hline Nitrate & $\begin{array}{l}\text { Isosorbide } \\
\text { mononitrate }\end{array}$ & COIDAI4 & $97(57.06)$ & 57.06 \\
\hline ACE inhibitor & Ramipril & C09AA05 & $85(50.00)$ & 50.00 \\
\hline Opioid analgesic & Tramadol & N02AX02 & $76(44.7 I)$ & 44.7I \\
\hline Stool softener & Liquid paraffin & A06AA0I & $68(40.00)$ & 40.00 \\
\hline $\begin{array}{l}\text { H2 receptor } \\
\text { blocker }\end{array}$ & Ranitidine & A02BA02 & 55 (32.35) & 32.35 \\
\hline Fibrinolytic & Streptokinase & BOIADOI & $48(28.24)$ & 28.24 \\
\hline Beta-blocker & Metoprolol & C07AB02 & $47(27.65)$ & 27.65 \\
\hline Antidiabetic & Insulin (regular) & AIOACOI & $45(26.47)$ & 26.47 \\
\hline
\end{tabular}

ATC: Anatomical therapeutic classification; PPI: Proton pump inhibitor; HMG: 3-hydroxy-3-methyl-glutaryl; 5-HT3: 5-hydroxy tryptamine3; ACE: Angiotensin converting enzyme; H2: Histamine. $n=170$

labetolol in one patient, which belonged to class $2 \mathrm{a}$ recommendations, all the other prescribed drugs were according to class 1 recommendations of ACC/ AHA guidelines. ${ }^{[9-11]}$ All the patients with STEMI received tramadol for analgesia instead of morphine, 